\title{
Refined parameters and spectroscopic transit of the super-massive planet HD 147506b^
}

\author{
B. Loeillet ${ }^{1,2}$, A. Shporer ${ }^{3}$, F. Bouchy ${ }^{2}$, F. Pont ${ }^{4}$, T. Mazeh ${ }^{3}$, J. L. Beuzit ${ }^{5}$, I. Boisse ${ }^{2}$, X. Bonfils ${ }^{5}$, R. Da Silva ${ }^{4}$, \\ X. Delfosse ${ }^{5}$, M. Desort ${ }^{5}$, A. Ecuvillon ${ }^{2}$, T. Forveille ${ }^{5}$, F. Galland ${ }^{5}$, A. Gallenne ${ }^{2}$, G. Hébrard ${ }^{2}$, A.-M. Lagrange , $^{5}$, \\ C. Lovis ${ }^{4}$, M. Mayor ${ }^{4}$, C. Moutou ${ }^{1}$, F. Pepe ${ }^{4}$, C. Perrier ${ }^{5}$, D. Queloz ${ }^{4}$, D. Ségransan ${ }^{4}$, J. P. Sivan ${ }^{1}$, N. C. Santos ${ }^{4,6}$, \\ Y. Tsodikovich ${ }^{3}$, S. Udry ${ }^{4}$, and A. Vidal-Madjar ${ }^{2}$
}

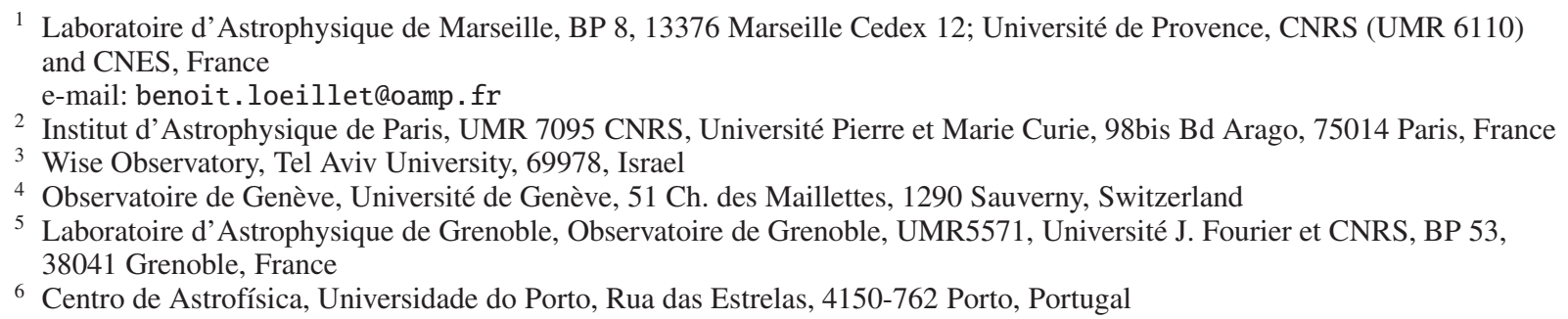

Received 26 June 2007 / Accepted 14 January 2008

\section{ABSTRACT}

\begin{abstract}
In this paper, we report a refined determination of the orbital parameters and the detection of the Rossiter-McLaughlin effect of the recently discovered transiting exoplanet HD 147506b (HAT-P-2b). The large orbital eccentricity at the short orbital period of this exoplanet is unexpected and is distinguishing from other known transiting exoplanets. We performed high-precision radial velocity spectroscopic observations of HD 147506 (HAT-P-2) with the new spectrograph SOPHIE, mounted on the $1.93 \mathrm{~m}$ telescope at the Haute-Provence observatory (OHP). We obtained 63 new measurements, including 35 on May 14 and 20 on June 11, when the planet was transiting its parent star. The radial velocity (RV) anomaly observed illustrates that HAT-P-2b orbital motion is set in the same direction as its parent star spin. The sky-projected angle between the normal of the orbital plane and the stellar spin axis, $\lambda=0.2_{-12.5}^{+12.2 \circ}$, is consistent with zero. The planetary and stellar radii were re-determined, yielding $R_{\mathrm{p}}=0.951_{-0.053}^{+0.039} R_{\mathrm{Jup}}, R_{\mathrm{s}}=1.416_{-0.062}^{+0.040} R_{\odot}$. The mass $\left(M_{\mathrm{p}}=8.62_{-0.55}^{+0.39} M_{\mathrm{Jup}}\right)$ and radius of HAT-P-2b indicate a density of $12.5_{-3.6}^{+2.6} \mathrm{~g} \mathrm{~cm}^{-3}$, suggesting an object in between the known close-in planets with typical density of the order of $1 \mathrm{~g} \mathrm{~cm}^{-3}$, and the very low-mass stars, with density greater than $50 \mathrm{~g} \mathrm{~cm}^{-3}$.
\end{abstract}

Key words. techniques: radial velocities - stars: individual: HD 147506 - stars: planetary systems

\section{Introduction}

Thirty of the almost 270 known extrasolar planets have been observed to transit their parent stars ${ }^{1}$. This small subgroup of planets have the highest impact on our overall understanding of close-in giant planets because we can estimate their mass and radius, and for some of them directly study their atmosphere. These transiting hot Jupiters have masses from 0.07 to about $8 M_{\text {Jup }}$ and radii from 0.3 to about $1.4 R_{\text {Jup }}$. This set of planets was recently extended to hot Neptune objects by the discovery of the transit of GJ436b (Gillon et al. 2007), and to super-massive planets by the detection of HD 147506b (HAT-P-2b) by Bakos et al. (2007) (hereafter B07). The discovery paper of the latter derived the key orbital and physical parameters of this exceptional object, which differs by its mass $\left(9.04 M_{\text {Jup }}\right)$, orbital period (5.63 days), and eccentricity (0.52) from other transiting planets. The radius of HD 147506b implies an uncommon measured mean density $\left(11.9 \mathrm{~g} \mathrm{~cm}^{-3}\right)$ and surface gravity $\left(227 \mathrm{~m} \mathrm{~s}^{-2}\right)$.

\footnotetext{
* Based on observations collected with the SOPHIE spectrograph on the 1.93-m telescope at OHP, France (programs 07A.PNP.MAZE and 07A.PNP.CONS).

${ }^{1}$ See http://obswww . unige.ch/ pont/TRANSITS.htm
}

These properties suggest that HAT-P-2b might be an intermediate object between giant planets and low-mass stars, near the brown dwarf population. Its density is indeed close to the upper limit of the planetary models (Baraffe et al. 2003) and may put this object in the transition region between planets and brown dwarfs.

We report here new RV measurements of HD 147506 (HAT-P-2) obtained to provide additional information and constraints on this unusual planetary system. This was done by 1) refining the orbital parameters, 2) refining the mass and radius of the companion and 3) measuring and modeling the Rossiter-McLaughlin effect (RV anomaly due to the partial eclipse of the rotating stellar surface). We present here a more precise orbital solution and the measurement of the sky projection of the inclination between the stellar spin axis and the normal of the orbital plane.

\section{Observations}

The parent star of HAT-P-2b, HD 147506, was observed in May and June 2007 with the new spectrograph SOPHIE (Bouchy $\&$ the Sophie team 2006) mounted on the 1.93-m telescope of Haute Provence Observatory. Observations were conducted in 
the high-efficiency mode (HE mode), which provides a spectral resolution of $R \sim 39000$. The Thorium-Argon lamp was used to calibrate the wavelength scale. The simultaneous ThAr calibration mode was not used, as wavelength calibration was performed less than $1 \mathrm{~h}$ before and after the observations leading to an instrumental stability of less than $2 \mathrm{~m} \mathrm{~s}^{-1}$.

We obtained eight out-of-transit spectra on six nights between May 6 and 15, 2007, and a sequence of $6 \mathrm{~h}$, comprised of 35 spectra during and after the transit on May 14 (JD = 2454235). Unfortunately, we could not observe the ingress phase of the transit that occurred just before twilight. Another sequence was obtained during transit on June 11 (JD = 2454263 ) to get a full coverage of the transit. The typical exposure time was $10 \mathrm{~min}$, long enough to reach a sufficiently high signal-tonoise ratio (SNR) and short enough to adequately sample the observed transit. The average SNR per pixel, at $\lambda=5500 \AA$, was about 70 during the first observed transit and about 90 during the second one. Another limitation of high-precision RV measurement in the mode used (HE) concerns the guidance centring of the target in the fiber. As the diameter of the fiber is quite large ( 3 arcsec), a strong decentring under very good seeing conditions $(<1.5$ arcsec $)$ could indeed induce a RV shift of a few tens $\mathrm{m} \mathrm{s}^{-1}$.

We determined RVs using a weighted cross-correlation method, following the procedure of Baranne et al. (1996) and Pepe et al. (2005), with a numerical mask constructed from the solar spectrum atlas corresponding to a G2 dwarf star. This standard mask is well adapted to the F8-type spectrum of the primary star. We estimated the measurement uncertainties based on the photon-noise empirical relation detailed by Bouchy et al. (2005) and Cameron et al. (2007). For the spectra obtained during the night of the first and the second transit, the typical photon-noise uncertainty is 14 and $10 \mathrm{~m} \mathrm{~s}^{-1}$, respectively, whereas for the other 8 spectra this uncertainty ranges from 6 to $28 \mathrm{~m} \mathrm{~s}^{-1}$. The journal of SOPHIE observations, including barycentric Julian dates (BJD), RVs, photon-noise uncertainties, and SNR per pixel is given in Table 1 . The phase-folded RVs are plotted in Fig. 1. As illustrated by the higher SNR values for the data acquired during the second night of observations, we have strong evidence that the observational conditions were very good. As explained above, the data are thus affected by guiding noise and we assumed an additional systematic error of $50 \mathrm{~m} \mathrm{~s}^{-1}$. We estimated this systematic error thanks to later instrumental tests under the same conditions.

\section{Stellar properties}

As described in B07, the spectroscopic determination of the radius of HD 147506 is very sensitive to the method used, as well as to the $\log g$ determination, which may be affected by the uncertainty of the spectrum continuum and the large projected rotational velocity, $v \sin I_{\mathrm{s}}$ of the star. The spectroscopic approach described in B07 provides a stellar radius of $1.474_{-0.167}^{+0.062} R_{\odot}$. Our combined SOPHIE spectrum provides an independent spectroscopic determination of the stellar parameters $\left(R_{\mathrm{S}}=1.416_{-0.062}^{+0.040} R_{\odot}\right)$, consistent with B07.

From the full width half maximum (FWHM) of the averaged cross-correlation functions (CCF) of SOPHIE spectra, which were calibrated to yield stellar $v \sin I_{\mathrm{s}}$ values (Santos et al. 2002), we determined the $v \sin I_{\mathrm{S}}$ of HD 147506 to be equal to $21.3 \pm$ $1.3 \mathrm{~km} \mathrm{~s}^{-1}$, somewhat slightly larger than the velocity derived by $\mathrm{B} 07\left(19.8 \pm 1.6 \mathrm{~km} \mathrm{~s}^{-1}\right)$. The metallicity index $[\mathrm{Fe} / \mathrm{H}]=$ $0.11 \pm 0.10$ we obtained, using the method described in Santos et al. (2002) is in full agreement with B07. The activity index of $\log R_{\mathrm{HK}}^{\prime}=-4.75 \pm 0.02$ was derived from the $\mathrm{H}$ and
Table 1. SOPHIE radial velocities of HD 147506 (HAT-P-2).

\begin{tabular}{|c|c|c|c|}
\hline $\begin{array}{c}\text { BJD } \\
-2400000\end{array}$ & $\begin{array}{c}\mathrm{RV} \\
{\left[\mathrm{km} \mathrm{s}^{-1}\right]}\end{array}$ & $\begin{array}{c}\text { Photon-noise } \\
\text { uncertainties } \\
{\left[\mathrm{km} \mathrm{s}^{-1}\right]}\end{array}$ & $\begin{array}{c}\text { Signal-to-noise ratio } \\
\text { per pixel } \\
\text { at } \lambda=5500 \AA\end{array}$ \\
\hline 54227.5016 & -19.4014 & 0.0088 & 109 \\
\hline 54227.6000 & -19.4082 & 0.0065 & 146 \\
\hline 54228.5842 & -19.5581 & 0.0188 & 54 \\
\hline 54229.5993 & -20.1874 & 0.0161 & 61 \\
\hline 54230.4475 & -21.2249 & 0.0141 & 68 \\
\hline 54230.6029 & -20.8536 & 0.0148 & 66 \\
\hline 54231.5987 & -19.5311 & 0.0121 & 78 \\
\hline 54235.3466 & -20.1916 & 0.0156 & 61 \\
\hline 54235.3538 & -20.2318 & 0.0180 & 53 \\
\hline 54235.3615 & -20.3008 & 0.0167 & 57 \\
\hline 54235.3692 & -20.2790 & 0.0173 & 55 \\
\hline 54235.3765 & -20.3083 & 0.0172 & 56 \\
\hline 54235.3866 & -20.3889 & 0.0209 & 46 \\
\hline 54235.3938 & -20.4280 & 0.0171 & 56 \\
\hline 54235.4011 & -20.4370 & 0.0173 & 55 \\
\hline 54235.4088 & -20.4450 & 0.0163 & 58 \\
\hline 54235.4161 & -20.4530 & 0.0180 & 53 \\
\hline 54235.4234 & -20.4987 & 0.0150 & 62 \\
\hline 54235.4310 & -20.5197 & 0.0144 & 65 \\
\hline 54235.4383 & -20.5032 & 0.0157 & 60 \\
\hline 54235.4456 & -20.5318 & 0.0178 & 53 \\
\hline 54235.4535 & -20.5340 & 0.0182 & 52 \\
\hline 54235.4608 & -20.4990 & 0.0143 & 66 \\
\hline 54235.4681 & -20.5047 & 0.0124 & 75 \\
\hline 54235.4759 & -20.4747 & 0.0117 & 81 \\
\hline 54235.4831 & -20.4974 & 0.0113 & 82 \\
\hline 54235.4904 & -20.5364 & 0.0111 & 84 \\
\hline 54235.4981 & -20.5384 & 0.0111 & 85 \\
\hline 54235.5054 & -20.5484 & 0.0108 & 87 \\
\hline 54235.5126 & -20.5785 & 0.0135 & 70 \\
\hline 54235.5204 & -20.6029 & 0.0142 & 66 \\
\hline 54235.5277 & -20.6066 & 0.0153 & 62 \\
\hline 54235.5350 & -20.5946 & 0.0115 & 81 \\
\hline 54235.5434 & -20.6155 & 0.0118 & 80 \\
\hline 54235.5507 & -20.5987 & 0.0113 & 82 \\
\hline 54235.5580 & -20.6266 & 0.0110 & 85 \\
\hline 54235.5682 & -20.6683 & 0.0119 & 80 \\
\hline 54235.5755 & -20.6712 & 0.0123 & 77 \\
\hline 54235.5827 & -20.6585 & 0.0115 & 82 \\
\hline 54235.5905 & -20.6819 & 0.0108 & 87 \\
\hline 54235.5978 & -20.7002 & 0.0128 & 74 \\
\hline 54235.6051 & -20.7205 & 0.0117 & 81 \\
\hline 54236.5190 & -20.2207 & 0.0056 & 99 \\
\hline 54263.4521 & -20.2050 & 0.0157 & 71 \\
\hline 54263.4594 & -20.1880 & 0.0100 & 102 \\
\hline 54263.4666 & -20.2048 & 0.0089 & 113 \\
\hline 54263.4739 & -20.1887 & 0.0092 & 110 \\
\hline 54263.4804 & -20.1626 & 0.0114 & 90 \\
\hline 54263.4860 & -20.1750 & 0.0120 & 86 \\
\hline 54263.4915 & -20.1832 & 0.0128 & 81 \\
\hline 54263.4971 & -20.1429 & 0.0096 & 104 \\
\hline 54263.5030 & -20.1565 & 0.0102 & 99 \\
\hline 54263.5086 & -20.1615 & 0.0108 & 95 \\
\hline 54263.5141 & -20.1610 & 0.0104 & 97 \\
\hline 54263.5236 & -20.1810 & 0.0103 & 99 \\
\hline 54263.5291 & -20.1955 & 0.0151 & 72 \\
\hline 54263.5347 & -20.3046 & 0.0179 & 63 \\
\hline 54263.5411 & -20.3391 & 0.0277 & 48 \\
\hline 54263.5561 & -20.3457 & 0.0127 & 85 \\
\hline 54263.5634 & -20.4009 & 0.0133 & 82 \\
\hline 54263.5706 & -20.4169 & 0.0173 & 67 \\
\hline 54263.5779 & -20.4377 & 0.0107 & 99 \\
\hline 54263.5852 & -20.4763 & 0.0091 & 113 \\
\hline
\end{tabular}




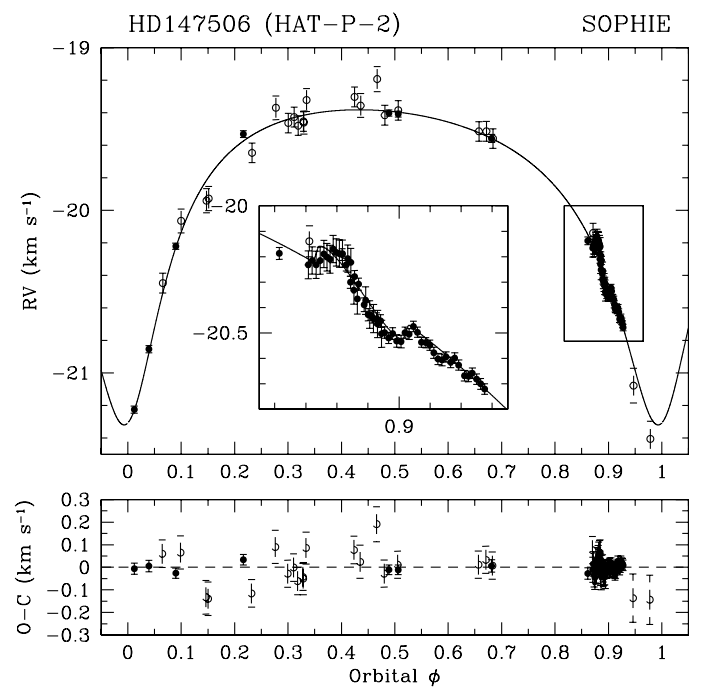

Fig. 1. Top: phase-folded Radial Velocity measurements of HAT-P-2 superimposed on the refined Keplerian orbital solution. Open circles refer to the Keck and Lick measurements. Filled circles refer to the SOPHIE measurements. The inset shows a zoom around the transit where radial velocities exhibit the Rossiter-McLaughlin effect. Bottom: residuals around the orbital solution.

$\mathrm{K}$ CaII lines and appears to be close to the value determined by B07 $\left(\log R_{\mathrm{HK}}^{\prime}=-4.72 \pm 0.05\right)$. As estimated in Santos et al. (2000), our activity index for a F8 dwarf star implies a stellar jitter from a few m s${ }^{-1}$ to about $20 \mathrm{~m} \mathrm{~s}^{-1}$, which is confirmed by the calibration made by Wright (2005). Analysis of the line-bisector computed for all out-of-transit spectra does not show significant variations nor correlation with the RVs.

\section{Determination of the planetary system parameters}

In this section, we first describe the procedure used to fit the observed velocities with the Keplerian orbit and the RM RV anomaly, and how we estimated the uncertainties. In the second and third subsections, we discuss our results.

\subsection{Analysis of the RV data}

We used all available high-precision spectroscopic data to model the orbit and the RM effect simultaneously. This data includes 10 Lick spectra and 13 Keck spectra obtained by B07, and the 63 SOPHIE spectra. Our model is comprised of 15 parameters: The period, $P$; periastron passage time, $T_{0}$; orbital eccentricity, $e$; angle between ascending node and periastron, $\omega$; RV semiamplitude, $K$; RV zero point, $V_{0}$ (these first six are the classical orbital parameters); planetary to stellar radii ratio, $R_{\mathrm{p}} / R_{\mathrm{s}}$; orbital semi-major axis to stellar radius ratio, $a / R_{\mathrm{s}}$; angle between sky projection of the orbital angular momentum axis and stellar spin axis, $\lambda$; line of sight stellar rotational velocity, $v \sin I_{\mathrm{s}}$; orbital inclination angle, $i$; and the stellar linear limb darkening coefficient, $\epsilon$. We have also determined a velocity shift between Keck and Lick velocity zero points, $\Delta v_{\mathrm{KL}}$, and SOPHIE and Keck zero points, $\Delta v_{\text {SK }}$. We also estimated a velocity shift, $\Delta v_{\mathrm{S} 2}$, between the SOPHIE measurements taken on the second transit night (June 11) and the rest of the SOPHIE measurements, taken about a month earlier. The period was fixed on the value given by B07 ( $P=5.63341$ days), considering its very high accuracy $(11 \mathrm{~s})$ derived from extensive photometric observations. A linear limb-darkening coefficient $\epsilon=0.71$ was also used, considering the stellar $T_{\text {eff }}=6250 \mathrm{~K}$ (Claret 2004). Hence, our model has 13 free parameters, where 8 are non-linear $\left(T_{0}, e, \omega, R_{\mathrm{p}} / R_{\mathrm{s}}, a / R_{\mathrm{s}}, \lambda, v \sin I_{\mathrm{s}}\right.$ and $\left.i\right)$ and 5 linear $\left(K, V_{0}, \Delta v_{\mathrm{KL}}, \Delta v_{\mathrm{SK}}\right.$ and $\left.\Delta v_{\mathrm{S} 2}\right)$.

We used a Keplerian model for the orbit, and the analytic approach described by Ohta et al. (2005), to model the RM effect. Equations given by Ohta et al. (2005) for the RM RV anomaly were modified to make them dependent on $R_{\mathrm{p}} / R_{\mathrm{S}}$ and $a / R_{\mathrm{S}}$, instead of $R_{\mathrm{p}}, R_{\mathrm{s}}$ and $a$.

We searched the parameter space for the global minimum $\chi^{2}$ position, using the equation below. We used the linear least square method, along with the data, to calculate the linear parameters at each position in the parameter space of the non-linear parameters. We modified our $\chi^{2}$ function to account for external information, namely the line-of-sight stellar rotational velocity, derived here from the spectra, the radii ratio, and transit duration, from B07:

$$
\begin{aligned}
\chi^{2}= & \Sigma_{i}\left(\frac{R V_{\mathrm{o}, i}-R V_{\mathrm{c}, i}}{R V_{\mathrm{err}, i}}\right)^{2}+\left(\frac{v \sin I_{\mathrm{s}}-21.3}{1.3}\right)^{2} \\
& +\left(\frac{R_{\mathrm{p}} / R_{\mathrm{s}}-0.0684}{0.0009}\right)^{2}+\left(\frac{T_{\mathrm{dur}}-0.177}{0.002}\right)^{2}
\end{aligned}
$$

where $R V_{\mathrm{o}, i}$ and $R V_{\mathrm{c}, i}$ are the $i$ th observed and calculated RVs and $R V_{\mathrm{err}, i}$ is its error. $T_{\mathrm{dur}}$ is the transit duration and is related to $a / R_{\mathrm{S}}, R_{\mathrm{p}} / R_{\mathrm{S}}, i$ and the orbital parameters $P, e$, and $\omega$. The uncertainties were computed directly from the linear least squares analysis for the linear parameters (Press et al. 1992, Sect. 15.4, Eq. (15.4.15)). The procedure is a bit more complex concerning the non-linear parameters. For each specific parameter, we increase and decrease it using small steps starting from the minimum $\chi^{2}$ solution value. At each step, the rest of the parameters are fitted while holding the specific parameter constant. Then, a 4 th degree polynomial is fitted to the $\chi^{2}$ values obtained and the $1-\sigma$ uncertainty is estimated by identifying the values of the fit corresponding to the minimum $\chi^{2}$ value +1 .

In the fit procedure, we adopted the B07 stellar jitter of $60 \mathrm{~m} \mathrm{~s}^{-1}$ for the Lick and Keck measurements. For the SOPHIE measurements, we adopted a jitter of $17 \mathrm{~m} \mathrm{~s}^{-1}$, resulting in a reduced $\chi^{2}=1$ for the out-of-transit SOPHIE measurements. This value is in a good agreement with the value estimated from our revised activity index and should be compared to the jitter of $60 \mathrm{~m} \mathrm{~s}^{-1}$ mentioned in B07, considering the span covered by Lick and Keck observations was 240 nights. Over such a long span, one cannot exclude variations in the stellar activity level. We also note that the effect of this jitter may have a time scale comparable or longer than the time of a single exposure, inducing a correlated noise effect.

\subsection{Results of the fitted orbital solution}

Table 2 lists the result for the fitted parameters of our model. The solution depends on the RV data and the prior constraints ( $v \sin I_{\mathrm{S}}, R_{\mathrm{p}} / R_{\mathrm{S}}$, transit duration), derived from the spectroscopic analysis in this work and the photometry from B07. The refined orbital solution is plotted in the upper panel of Figs. 1, and 2 presents the RM RV anomaly model after subtraction of the Keplerian orbit.

The rms of the out-of-transit residuals of SOPHIE measurements, spanning 36 days, is equal to $18 \mathrm{~m} \mathrm{~s}^{-1}$, whereas rms of all out-of-transit measurements, spanning 282 days, is $50 \mathrm{~m} \mathrm{~s}^{-1}$. The orbital parameters we derived are consistent with B07. 
Table 2. Refined parameters of the Keplerian orbital solution and the parameters of the HAT-P-2 system, compared to those determined in (Bakos et al. 2007, B07). $T_{0}$ refers to the periastron time. The fitted parameters are presented at the top of the table. The bottom part of the table lists the system parameters, derived from the fitted ones. We present in the table both the spectroscopic determination and the result from the fit of the star's $v \sin I_{\mathrm{s}}$.

\begin{tabular}{|c|c|c|}
\hline Parameter & Value & Value from B07 \\
\hline Period $[\mathrm{d}]^{\star}$ & 5.63341 (fixed) & $5.63341 \pm 0.00013$ \\
\hline$T_{0}[\mathrm{HJD}]$ & $2454213.47944_{-0.0030}^{+0.0053}$ & $2454213.369 \pm 0.041$ \\
\hline$e$ & $0.5163_{-0.0023}^{+0.0025}$ & $0.520 \pm 0.010$ \\
\hline$\omega[\mathrm{deg}]$ & $189.92_{-1.20}^{+0.0023}$ & $179.3 \pm 3.6$ \\
\hline$K\left[\mathrm{~m} \mathrm{~s}^{-1}\right]$ & $966.9 \pm 8.3$ & $1011 \pm 38$ \\
\hline$V_{0}\left[\mathrm{~m} \mathrm{~s}^{-1}\right]$ & $-19855.1 \pm 5.8$ & N.A. \\
\hline$R_{\mathrm{p}} / R_{\mathrm{s}}$ & $0.06891_{-0.00086}^{+0.00090}$ & $0.0684 \pm 0.0009$ \\
\hline$a / R_{\mathrm{s}}$ & $10.28_{-0.19}^{+0.12}$ & $9.77_{-0.02}^{+1.10}$ \\
\hline$\lambda[\mathrm{deg}]$ & $0.2_{-12 .}^{+12.2}$ & N.A. \\
\hline$v \sin I_{\mathrm{S} \mathrm{RM}}\left[\mathrm{km} \mathrm{s}^{-1}\right]$ & $22.9_{-1.2}^{+1.3}$ & N.A. \\
\hline$v \sin I_{\mathrm{s}}$ Spectro $\left[\mathrm{km} \mathrm{s}^{-1}\right]$ & $21.3^{-1.2} \pm 1.3$ & $19.8 \pm 1.6$ \\
\hline$i[\mathrm{deg}]$ & $90.0_{-0.93}^{+0.85}$ & $>84.6(2 \sigma)$ \\
\hline$\Delta v_{\mathrm{KL}}\left[\mathrm{m} \mathrm{s}^{-1}\right]$ & $-328 \pm 41$ & $-380 \pm 35$ \\
\hline$\Delta v_{\mathrm{SK}}\left[\mathrm{m} \mathrm{s}^{-1}\right]$ & $-19584 \pm 17$ & N.A. \\
\hline$\Delta v_{\mathrm{S} 2}\left[\mathrm{~m} \mathrm{~s}^{-1}\right]$ & $-27 \pm 12$ & N.A. \\
\hline Stellar jitter $\left[\mathrm{m} \mathrm{s}^{-1}\right]^{\star \star}$ & 17 & 60 \\
\hline$[\mathrm{Fe} / \mathrm{H}](\mathrm{dex})$ & $0.11 \pm 0.10$ & $0.12 \pm 0.08$ \\
\hline $\log R_{\mathrm{HK}}^{\prime}$ & $-4.75 \pm 0.02$ & $-4.72 \pm 0.05$ \\
\hline$M_{\mathrm{s}}\left[M_{\odot}\right]^{\star}$ & $1.298_{-0.098}^{+0.062}$ & $1.298_{-0.098}^{+0.062}$ \\
\hline$a[\mathrm{AU}]$ & $0.0677_{-0.0017}^{+0.098}$ & $0.0677 \pm 0.0014$ \\
\hline$R_{\mathrm{s}}\left[R_{\odot}\right]$ & $1.416_{-0.062}^{+0.04}$ & $1.474_{-0.0167}^{+0.042}$ \\
\hline$M_{\mathrm{p}}\left[M_{\mathrm{Jup}}\right]$ & $8.62_{-0.55}^{+0.0062}$ & $9.04 \pm 0.50$ \\
\hline$R_{\mathrm{p}}\left[R_{\mathrm{Jup}}\right]$ & $0.951_{-0.053}^{-0.55}$ & $0.982_{-0105}^{+0.038}$ \\
\hline$\rho_{\mathrm{p}}\left[\mathrm{g} \mathrm{cm}^{-3}\right]$ & $12.5_{-3.6}^{+2.0 .0 .}$ & $11.9_{-16}^{+4.8}$ \\
\hline$g_{\mathrm{p}}\left[\mathrm{m} \mathrm{s}^{-2}\right]$ & $237_{-41}^{+30.0}$ & $227_{-16}^{+4.0}$ \\
\hline
\end{tabular}

${ }^{*}$ Adopted from B07; ${ }^{* *}$ short term jitter.

However our uncertainties are smaller, due to a larger sample of high-precision RV data.

We searched for a second planetary signal in the RV residuals to look for hints of a third body in the system. No clear periodic signal appears in the RV residuals. We estimated that we can exclude the presence of a second planet of mass greater than 1.3, 1.5 and $1.8 M_{\text {Jup }}$ for an orbital period shorter than 50, 100, and 200 days respectively. However, the increased RMS for all outof-transit residuals suggests that a long-term RV follow-up of this star is needed.

\subsection{Measurement of the Rossiter-McLaughlin effect}

As an important result, the sign of the RV anomaly shows that the orbital motion is set in the same direction as the stellar spin, similar to the four previous observed RM effects on transiting exoplanets (Queloz et al. 2000; Winn et al. 2005, for HD 209458; Winn et al. 2006, for HD 189733; Wolf et al. 2007, for HD 149026; and Narita et al. 2007, for TrES-1). The orbital inclination angle $i$ we derived is in full agreement with the value determined by $\mathrm{B} 07$. The projected rotation velocity of the star $v \sin I_{\mathrm{S}}$ determined from the RM fit is $22.9 \pm 1.2 \mathrm{~km} \mathrm{~s}^{-1}$. This value is greater than our spectroscopic determination from SOPHIE CCFs, and 2- $\sigma$ greater than the determination of B07. However, Winn et al. (2005) showed that $v \sin I_{\mathrm{S}}$ measured with the analytical formulae from Ohta et al. (2005) is biased toward

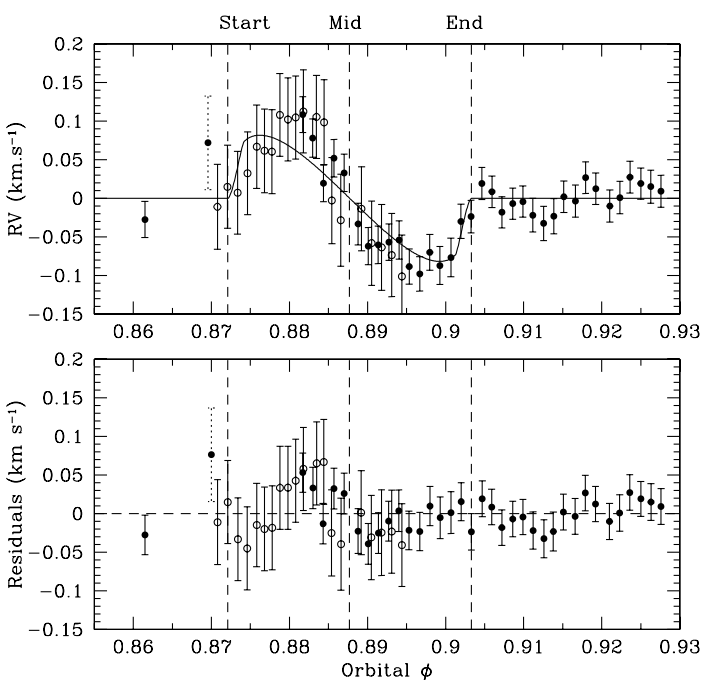

Fig. 2. Top: radial velocities of HAT-P-2 as a function of the orbital phase after subtraction of the Keplerian model and superimposed with the best fit of the Rossiter-McLaughlin effect. The filled and open circles represent the RV measurements obtained during the first and the second sequence of observations of the transit with SOPHIE, respectively. The open circle with dotted error bars represent one measurement from the Keck set which is a few minutes before the ingress phase. Bottom: RV residuals after subtracting the orbital solution and the modeled RM effect.

larger velocities by approximately $10 \%$. Moreover, as the planet crosses the star at the equatorial plane, assuming a differential rotational velocity of the star, the fitted $v \sin I_{\mathrm{s}}$ corresponds to the maximum value. Küker \& Rüdiger (2005) show that a differential rotational rate can be as high as about $10 \%$ for a F8-type star. The projected angle between the stellar spin axis and the normal of the orbital plane, $\lambda=0.2 \pm 12.5^{\circ}$, is consistent with complete alignment of the stellar spin and orbital angular momentum.

We extended our analysis in the search for the global minimum $\chi^{2}$, without any constraint on the $v \sin I_{\mathrm{s}}$. The result obtained gives a significantly larger $v \sin I_{\mathrm{s}}$ value $\left(29.5_{-2.2}^{+3.1} \mathrm{~km} \mathrm{~s}^{-1}\right)$ and a consistent lambda value $\left(5.0_{-6.0}^{+17.8 \circ}\right)$. However, such a $v \sin I_{\mathrm{s}}$ value is clearly incompatible with the spectroscopic determination of the rotational velocity of the star.

We also computed the line-bisector behavior following the procedure described in Santos et al. (2002). As shown in Fig. 3, a specific signature of the line-bisectors can be found during the transit and is anti-correlated with the RVs due to the fact that the crossing planet mainly affects the bottom of the spectral lines.

Assuming the B07 stellar mass and period, of $1.298_{-0.098}^{+0.062} M_{\odot}$ and 5.63341 days, and using the fitted ratios $R_{\mathrm{p}} / R_{\mathrm{s}}$ and $a / R_{\mathrm{s}}$, we also provide a new determination of the system parameters. Those include: orbital semi-major axis, $a$, stellar radius, $R_{\mathrm{s}}$, and the planetary radius, $R_{\mathrm{p}}$, mass, $M_{\mathrm{p}}$, density $\rho_{\mathrm{p}}$, and surface gravity $g_{\mathrm{p}}$. Our results are similar to those of B07 with smaller errors. The RVs describing the spectroscopic transit are quite noisy and some of the measured RVs (around orbital phase 0.885) present an unexpected RV shift. This shift does not seem to be due to an instrumental deviation and may be explained by a guidance decentring of the telescope. However, the amplitude of the Rossiter anomaly is large enough that we can still estimate the parameters of this system with quite good uncertainties. 


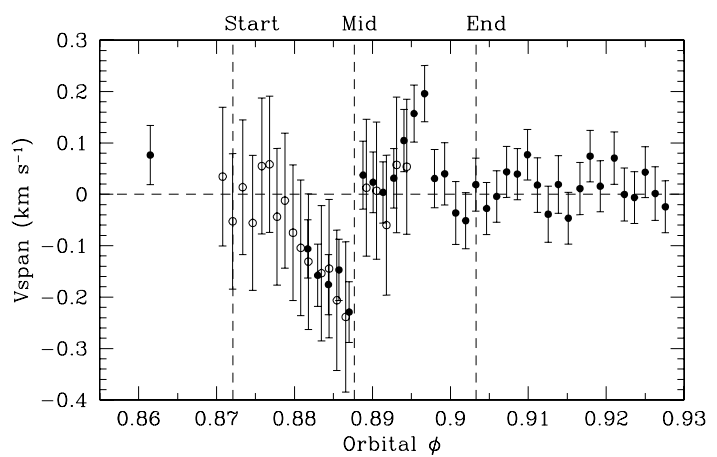

Fig. 3. Line-bisectors signature during the spectroscopic transit as a function of the orbital phase. As in Fig. 2 the filled and open circles represent the RV measurements obtained during the first and second sequences of transit observations with SOPHIE, respectively.

\section{Discussion and conclusion}

The value of HAT-P-2b radius $\left(0.95 R_{\text {Jup }}\right)$ puts this object in the mass-radius diagram as an intermediate case between HotJupiters and low-mass stars. Its mean density of $12.5 \mathrm{~g} \mathrm{~cm}^{-3}$ is in between the Hot-Jupiter density $\left(0.34-1.34 \mathrm{~g} \mathrm{~cm}^{-3}\right)$ and the density of the smallest transiting M dwarfs OGLE-TR-122 and OGLE-TR-123 (Pont et al. 2005, 2006), which are 75 and $51 \mathrm{~g} \mathrm{~cm}^{-3}$, respectively. A similar paper (Winn et al. 2007) published results that are consistent with our conclusions. Supermassive planets like HAT-P-2b may constitute a new class of stellar companions, in between Hot-Jupiters and low-mass stars and near the Brown dwarf population. HAT-P-2b is the first super-massive object around a F8 star for which the exact mass has been determined. Such a massive close-in planet is not in agreement with the type II migration mechanism, which appears to be more efficient for planets around low-mass stars (Ida \& Lin 2005). This could suggest a different formation process for this object, such as fragmentation, or interactions between the planet and another companion or between the planet and the disk in the evolution process.

A system with an elliptic orbit is expected to move toward pseudo-synchronization, with the stellar angular rotation velocity tuned to near the angular velocity of the companion at periastron passage (Zahn 1977). Given the Keplerian orbital parameters, we computed the angular planetary speed at the periastron position and found that a stellar radius of $1.42 R_{\odot}$ implies a pseudo-synchronization rotational velocity $v \sin I_{\mathrm{s}}$ of about $40 \mathrm{~km} \mathrm{~s}^{-1}$ (Hut 1981, Eq. (43)). Because the observed rotational velocity of the star is only about $21 \mathrm{~km} \mathrm{~s}^{-1}$, the star is definitely not pseudo-synchronized to the planetary orbit. Peale (1999) indicated that the alignment and the synchronization timescales are of the same order of magnitude. Therefore, the lack of pseudosynchronization indicates that the system was formed with the stellar spin aligned to the orbital angular momentum.

Acknowledgements. Part of these observations have been funded by the Optical Infrared Coordination network (OPTICON), a major international collaboration supported by the Research Infrastructures Programme of the European Commission's Sixth Framework Programme. N.C.S. would like to thank the support from Fundao para a Cincia e a Tecnologia, Portugal, in the form of a grant (reference POCI/CTE-AST/56453/2004). This work was supported in part by the EC's FP6 and by FCT (with POCI2010 and FEDER funds), within the HELAS international collaboration. A.E. would like to thank the support from the Swiss National Science Foundation (SNSF), Switzerland, in the form of a grant (reference PBSK2-114688). We thank the technical team from OHP who worked on the instrument SOPHIE and for their exceptional work.

\section{References}

Bakos, G. A., Kovacs, G., Torres, G., et al. 2007, ArXiv e-prints, 705 Baraffe, I., Chabrier, G., Barman, T. S., Allard, F., \& Hauschildt, P. H. 2003, A\&A, 402, 701

Baranne, A., Queloz, D., Mayor, M., et al. 1996, A\&AS, 119, 373

Bouchy, F., Pont, F., Melo, C., et al. 2005, A\&A, 431, 1105

Bouchy, F., \& The Sophie Team 2006, in Tenth Anniversary of 51 Peg-b: Status of and prospects for hot Jupiter studies, ed. L. Arnold, F. Bouchy, \& C. Moutou, 319

Cameron, A. C., Bouchy, F., Hébrard, G., et al. 2007, MNRAS, 375, 951

Claret, A. 2004, A\&A, 428, 1001

Gillon, M., Pont, F., Demory, B., et al. 2007, ArXiv e-prints, 705

Hut, P. 1981, A\&A, 99, 126

Ida, S., \& Lin, D. N. C. 2005, ApJ, 626, 1045

Küker, M., \& Rüdiger, G. 2005, A\&A, 433, 1023

Narita, N., Enya, K., Sato, B., et al. 2007, ArXiv Astrophysics e-prints

Ohta, Y., Taruya, A., \& Suto, Y. 2005, ApJ, 622, 1118

Peale, S. J. 1999, ARA\&A, 37, 533

Pepe, F., Mayor, M., Queloz, D., et al. 2005, The Messenger, 120, 22

Pont, F., Bouchy, F., Melo, C., et al. 2005, A\&A, 438, 1123

Pont, F., Moutou, C., Bouchy, F., et al. 2006, A\&A, 447, 1035

Press, W. H., Teukolsky, S. A., Vetterling, W. T., \& Flannery, B. P. 1992,

Numerical recipes in C. The art of scientific computing, 2nd Ed. (Cambridge: University Press)

Queloz, D., Eggenberger, A., Mayor, M., et al. 2000, A\&A, 359, L13

Santos, N. C., Mayor, M., Naef, D., et al. 2000, A\&A, 361, 265

Santos, N. C., Mayor, M., Naef, D., et al. 2002, A\&A, 392, 215

Winn, J. N., Noyes, R. W., Holman, M. J., et al. 2005, ApJ, 631, 1215

Winn, J. N., Johnson, J. A., Marcy, G. W., et al. 2006, ApJ, 653, L69

Winn, J. N., Johnson, J. A., Peek, K. M. G., et al. 2007, ApJ, 665, L167

Wolf, A. S., Laughlin, G., Henry, G. W., et al. 2007, ApJ, 667, 549

Wright, J. T. 2005, PASP, 117, 657

Zahn, J.-P. 1977, A\&A, 57, 383 